\title{
ARTICLES
}

\section{Newtonian glass fiber drawing: Chaotic variation of the cross-sectional radius}

\author{
A. L. Yarin \\ Faculty of Mechanical Engineering, Technion-Israel Institute of Technology, Haifa 32000, Israel \\ and Chemical Engineering Department and Rheology Research Center, University of Wisconsin-Madison, \\ Madison, Wisconsin 53706-1691 \\ P. Gospodinov \\ Institute of Mechanics, Bulgarian Academy of Sciences, Acad. G. Bonchev St., Bl. 4, Sofia 1113, Bulgaria \\ O. Gottlieb \\ Faculty of Mechanial Engineering, Technion-Israel Institute of Technology, Haifa 32000, Israel \\ M. D. Graham \\ Chemical Engineering Department and Rheology Research Center, University of Wisconsin-Madison, \\ Madison, Wisconsin 53706-1691
}

(Received 15 April 1998; accepted 21 July 1999)

A model of Newtonian glass fiber drawing at fixed temperature in the unsteady range (the draw ratio $E>20.22$ ) is considered. In this range under steady boundary conditions, as is well known, the draw resonance instability sets in, resulting in self-sustained oscillations. These oscillations lead to a periodic variation of the cross-sectional radius of the fiber. In the present work we consider the case where the spinline radius varies periodically. Such a variation may result from flow oscillations in the fiber forming channels in the direct-melt process, or from the variation of the preform cross-sectional radius in drawing of optical fibers. When this variation takes place in the range $E$ $>20.22$, the self-sustained periodic oscillations of the draw resonance are replaced by quasiperiodic and periodic (mode-locked) subharmonic or (under the appropriate conditions) chaotic oscillations (strange attractors). The routes to chaos found in the present work include (1) smooth period doubling bifurcation of (any) mode-locked periodic solution, (2) abrupt explosions of quasiperiodic solutions. The predicted chaotic variation of the spinline radius at the winding mandrel may result in a similar variation of the cross-sectional radius of the solidified fibers. (C) 1999 American Institute of Physics. [S1070-6631(99)01411-7]

\section{INTRODUCTION}

Glass fibers are used for air filtration, thermal and electrical insulation, plastics reinforcement, for manufacturing the glass textile products, optical communications, etc. ${ }^{1,2}$ In the direct-melt processes fibers are formed by drawing molten glass from heated furnaces (sometimes called "bushings"), whereas optical fibers are drawn from solid preforms heated in a furnace or directly by laser beams. ${ }^{1-4}$ The preforms are created using a modified chemical vapor deposition process (MCVD) (Ref. 5) or a nonsymmetric deposition process (N-MCVD). ${ }^{6}$

Molten glass is a Newtonian fluid with a strong dependence of viscosity on temperature. Drawing of glass fibers is typically dominated by viscous forces modified by heating and cooling of the threadline. Under appropriate conditions perturbations introduced in a molten threadline lead to an instability referred to as draw resonance. ${ }^{7-9}$ It sets in both isothermal and nonisothermal cases. In Ref. 7 it was shown that in the former case the draw resonance sets in when the draw ratio $E=V_{1} / V_{0}$ exceeds a value close to $20\left(V_{0}\right.$ and $V_{1}$ are the input and the output velocities in the threadline).

In Ref. 8 it was shown that the instability evolves to a stable limit-cycle solution: periodic self-sustained oscillations, e.g., of the cross-sectional radius of the drawn fiber. The instability manifests itself in the form of propagating waves of swelling and contraction of the cross section with a wavelength of the order of the threadline length.

In Refs. 10 and 11 it was shown that the bifurcation at $E=20.22$ corresponding to the isothermal draw resonance is supercritical. Further bifurcations at higher $E$ were not found (including the cases with nonisothermal spinning conditions ${ }^{9,12-15}$ ), which means that the structure of the draw resonance instability as a Hopf bifurcation survives even under nonisothermal conditions. Heat removal stabilizes the spinning process leading to an increase (sometimes significant) in the threshold value of the draw ratio which can become much larger than 20.22 predicted for the isothermal case. ${ }^{9,12-15}$ The theoretical findings on the heat removal effect on the draw resonance instability are fully supported by experimental data. ${ }^{16,17}$ Also an isothermal drawing of New- 
tonian liquids was realized in model experiments ${ }^{8,18}$ which showed, in agreement with the predictions, that the instability sets in as soon as the draw ratio reaches a value close to $E=20$.

It is emphasized that the threshold value $E=20.22$ was obtained theoretically in the framework of the quasi-onedimensional description of the molten threadline. ${ }^{7-9,15} \mathrm{Com}-$ parison of the theoretical and experimental data in Refs. 9 and 15 showed that a simple quasi-one-dimensional description is a good approximation for both direct-melt and even laser drawn processes. It is also known to be very accurate in description of other longwave phenomena and instabilities in free liquid jets and films of Newtonian and non-Newtonian liquids. ${ }^{15,19-23}$

In the above-mentioned stability studies boundary conditions for the molten threadline were fixed, and the draw resonance instability set in and developed itself into a limit cycle only due to the on-line perturbations. A possible variation of the input parameters and winding velocity was accounted for only in the range $E<20.22$ in the previous publications to study fiber sensitivity to the external perturbations. ${ }^{7,14,15}$ In reality, however, variation of the input parameters and take-up velocity is inevitable also in the supercritical regimes. ${ }^{3}$ Oscillations of the input parameters in glass fiber drawing are related to the processes taking place in the tank furnace. Considerable thermal inertia of the electric furnaces makes temperature stabilization difficult, which causes oscillations of the boundaries of the melting zone. This, in turn, results in flow oscillations in the fiber forming channels and oscillations of the threadline radius at the channel exit. Changes in composition of the glass, loading of glass marbles by a local feeder during some limited time intervals, etc. have a similar effect. In optical fiber drawing similar perturbations can be introduced by a nonuniform distribution of the cross-sectional radius over a solid preform. In practice it is important to understand the reason and the nature of the perturbations, since this information can be used to develop sensors for controlling the thickness of glass fiber during production by means of automatically regulating, for example, the temperature in the electric furnace according to the thickness of the glass thread.

The results of several experimental investigations (e.g., Ref. 24, and references therein) show ".. that a change of thickness of glass fiber length-wise is a random process..." and the corresponding correlation function decays exponentially. Draw resonance, being a periodic process, cannot be responsible for this picture. A possible source for the random unevenness of drawn fibers may be random noise entering a threadline. This, however, should lead to random perturbations for any drawing speeds, which is not the case; they are seen at the fibers drawn only at high enough speed (cf., e.g., Ref. 3). In the present work we explore another possibility and show that randomness may be generic for fiber drawing with high enough speeds characteristic of the draw resonance in the case when additional periodic perturbations enter the threadline from the channel/nozzle or a preform. To show that this scenario is, in principle, possible, we use the simplest Newtonian isothermal quasi-one-dimensional model of threadline. We show that a nonlinear interplay between the periodic perturbations of the input radius of the threadline and the draw resonance instability may lead to evolution of the limit-cycle solution to periodic (ultrasubharmonic) solutions and quasiperiodic tori which themselves bifurcate resulting in strange attractors corresponding to chaotic variation of the output radius (cf. Ref. 25).

In Sec. II the problem is formulated, and some known relevant results of linear stability analysis are presented in Sec. III. The bifurcation structure of the nonlinear problem is obtained numerically and presented in Sec. IV. Concluding remarks are given in Sec. V.

\section{PROBLEM FORMULATION}

The quasi-one-dimensional equations of continuity and momentum balance read, respectively, $7,9,1,26$

$$
\begin{aligned}
& \frac{\partial a^{2}}{\partial t}+\frac{\partial a^{2} V}{\partial x}=0, \\
& \rho a^{2}\left(\frac{\partial V}{\partial t}+V \frac{\partial V}{\partial x}\right)=\frac{\partial}{\partial x}\left(3 \mu a^{2} \frac{\partial V}{\partial x}\right)+\rho g a^{2}+\sigma \frac{\partial a}{\partial x} .
\end{aligned}
$$

Here $t$ is time, $x$ is the longitudinal coordinate along the fiber axis, $a$ is the cross-sectional radius (the fiber is assumed to be axisymmetric), $V$ is the longitudinal velocity, $\rho$ is the density, $\mu$ is the viscosity (the flow is considered to be isothermal and the fluid is assumed to be Newtonian with a constant viscosity; $3 \mu$ is the Trouton viscosity), $\sigma$ is the surface tension, $g$ is the gravity acceleration. In the last term in (2) it is also assumed that $a, x^{2} \ll 1$ which is a plausible approximation for glass fiber drawing. The momentum Eq. (2) incorporates all the relevant effects, namely, the inertial, viscous, gravitational, as well as those due to surface tension.

The equations are rendered dimensionless by the following scales: the threadline length $L$-for $x$, the take-up velocity $V_{1}$-for $V, L / V_{1}$-for $t$, and $a_{0} / E^{1 / 2}$-for $a$ (where $a_{0}$ is the unperturbed upstream radius of the threadline). As a result, the dimensionless continuity equation keeps the same form as in (1), whereas the momentum equation takes the following form:

$$
C_{1} a^{2}\left(\frac{\partial V}{\partial t}+V \frac{\partial V}{\partial x}\right)=\frac{\partial}{\partial x}\left(a^{2} \frac{\partial V}{\partial x}\right)+C_{2} a^{2}+C_{3} \frac{\partial a}{\partial x},
$$

where

$$
\begin{aligned}
& C_{1}=\frac{\operatorname{Re}}{3} \cdot \frac{L}{a_{0}}, \quad C_{2}=\frac{\operatorname{Re}}{3 \cdot \operatorname{Fr}}\left(\frac{L}{a_{0}}\right)^{2}, \\
& C_{3}=\frac{\operatorname{Re} \cdot E^{1 / 2}}{3 \cdot \mathrm{We}} \cdot \frac{L}{a_{0}}, \\
& \operatorname{Re}=\frac{\rho a_{0} V_{1}}{\mu}, \quad \mathrm{We}=\frac{\rho V_{1}^{2} a_{0}}{\sigma}, \quad \mathrm{Fr}=\frac{V_{1}^{2}}{g a_{0}},
\end{aligned}
$$

and bars over the dimensionless variables are dropped.

The values of the parameters involved are as follows: $\rho=3000 \mathrm{~kg} / \mathrm{m}^{3}, \sigma=0.25 \mathrm{~N} / \mathrm{m}, \mu=10^{5} \mathrm{Pas}$ (which corresponds to an optical fiber glass at the temperature about 1000 $\mathrm{K})$, take-up velocity of the order of $V_{1}=0.05 \mathrm{~m} / \mathrm{s}(3 \mathrm{~m} / \mathrm{min})$, initial fiber radius $a_{0}=0.005 \mathrm{~m}$, and the threadline length $L$ 
$=0.15 \mathrm{~m}$. The data correspond to the device and processing parameters described in detail in Ref. 27. The corresponding values of the Reynolds, Weber, and Froude numbers are as follows: $\operatorname{Re}=7.5 \cdot 10^{-6}, \mathrm{We}=0.15$, and $\mathrm{Fr}=0.051$. Also $L / a_{0}=30$. Therefore the parameters $C_{1}-C_{3}$ in (3)-(5) have the following values: $C_{1}=7.5 \cdot 10^{-5}, C_{2}=0.0441$, and $C_{3}$ $=0.00224$ (for $E=20$ ). These estimates unambiguously show that in the optical fiber drawing processes of interest in the present work the effects of inertia, gravity, and surface tension may be neglected as compared to the viscous term (the first one on the right in (3)). Indeed, the inertial term is of the order of $10^{-4}$, the gravity term $O\left(10^{-2}\right)$, the surface tension term $O\left(10^{-3}\right)$, whereas the viscous term $O(1)$. Such a conclusion is also supported by Ref. 28 , where a possible capillary instability of molten threadlines due to surface tension is attributed only to overheated liquids with low flow velocity - the regimes which could (and should) always be avoided in practice. At much higher velocities than those of interest here, e.g., characteristic of high-speed drawing of glass or polymer fibers with the take-up velocities of the order of $10^{3} \mathrm{~m} / \mathrm{min}$ (Refs. 2 and 29), inertial effects cannot be neglected in the momentum equation, and the air drag force should be incorporated. These regimes are not in the scope of the present work, where in the light of the above estimates, all the terms in the momentum equation (3) containing the factors $C_{1}-C_{3}$ can be in principle neglected. However, in the numerical simulations of Sec. IV all these terms are accounted for. Their effect was checked and proven once more (as, e.g., in Ref. 23) to be minor under the conditions of interest in the present work.

\section{BOUNDARY CONDITIONS AND RELEVANT LINEAR RESULTS}

In the steady state a solution of the dimensionless equations is subject to the following boundary conditions:

$$
\begin{aligned}
& x=0: \quad a=E^{1 / 2}, \quad V=E^{-1}, \\
& x=1: \quad V=1,
\end{aligned}
$$

which means that the input cross-sectional radius and velocity are given, together with the take-up velocity.

The analytical linear analysis of the small perturbations is possible only in the case where the secondary effects related with the inertial, gravitational, and surface tension forces are neglected completely (otherwise the analysis may be done only numerically, see Sec. IV). Since the physical estimates presented in Sec. II justify this in the case under consideration, a viscosity dominated version of the momentum Eq. (3) emerges, namely,

$$
\frac{\partial}{\partial x}\left(a^{2} \frac{\partial V}{\partial x}\right)=0
$$

together with the continuity Eq. (1) (both dimensionless).

The steady-state solution of these equations reads

$$
a_{s}=E^{(1 / 2)(1-x)}, V_{s}=E^{x-1},
$$

where subscript $s$ stands for "steady."

The perturbed solution of (1) and (9) was found in Ref. 7 in the following form:
TABLE I. Spectrum (15) of the linearized stability problem.

\begin{tabular}{lll}
\hline \hline \multicolumn{1}{c}{$E$} & \multicolumn{1}{c}{$\Lambda_{r}$} & \multicolumn{1}{c}{$\Lambda_{i}$} \\
\hline 19 & -0.0111 & 0.731 \\
20.22 & 0 & 0.693 \\
21 & 0.00495 & 0.6723 \\
25 & 0.01918 & 0.5869 \\
28 & 0.0258 & 0.5369 \\
30 & 0.029 & 0.508 \\
35 & 0.0349 & 0.4497 \\
\hline \hline
\end{tabular}

$$
a=a_{s}(1+\alpha), \quad V=V_{s}(1+\beta),
$$

where $\alpha$ and $\beta$ are the small perturbations subject to the following boundary conditions:

$$
\begin{array}{ll}
x=0: & \alpha=0, \quad \beta=0, \\
x=1: & \beta=0 .
\end{array}
$$

The linearized solution for $\alpha$ reads $^{7}$

$$
\alpha=e^{\Lambda t} F(x), \quad F(x)=\int_{0}^{x} \exp \left(\frac{\Lambda E^{1-x}}{\ln E}\right) d x,
$$

where the eigenvalue $\Lambda$ can be found from the following spectral equation:

$$
\Lambda=-\frac{F(1)}{\int_{0}^{1} E^{1-x} F(x) d x} .
$$

At $E<20.22$ the spectral Eq. (15) possesses only the solutions with negative real part $\Lambda_{r}$, defining asymptotically stable solutions (established first in Ref. 7). At $E=20.22$ the nondecaying small oscillations set in corresponding to the solution of (15) $\Lambda \approx i \cdot 0.693$, where $i$ is the imaginary unit. This is a classical Hopf bifurcation. At $20.22<E<49.98$ the solutions of (15) include a pair of complex conjugate solutions with positive real part $\Lambda_{r}$. The corresponding linear perturbations grow in time, and the draw resonance sets in. Some of the relevant eigenvalues found from (15) are listed in Table I, where $\Lambda_{i}$ denotes the imaginary part of $\Lambda$.

At $E>20.22$, the instability of the steady solution (10) results in a bifurcation to a new solution. Under the fixed boundary conditions it represents itself the self-sustained oscillations (a limit-cycle solution) illustrated by the numerical results depicted in Fig. 1 which is discussed in more detail in the next section.

In the present work, however, we allow for a periodic variation of the input fiber radius as discussed above, so that (7) and (8) are replaced by the following boundary conditions:

$$
\begin{aligned}
& x=0: \quad a=E^{1 / 2}(1+\delta \cos \omega t), \quad V=E^{-1}, \\
& x=1: \quad V=1,
\end{aligned}
$$

with $\delta$ and $\omega$ being the perturbation amplitude and frequency.

In the present work we study numerically nonlinear solutions of the governing Eqs. (1) and (3) subject to the 

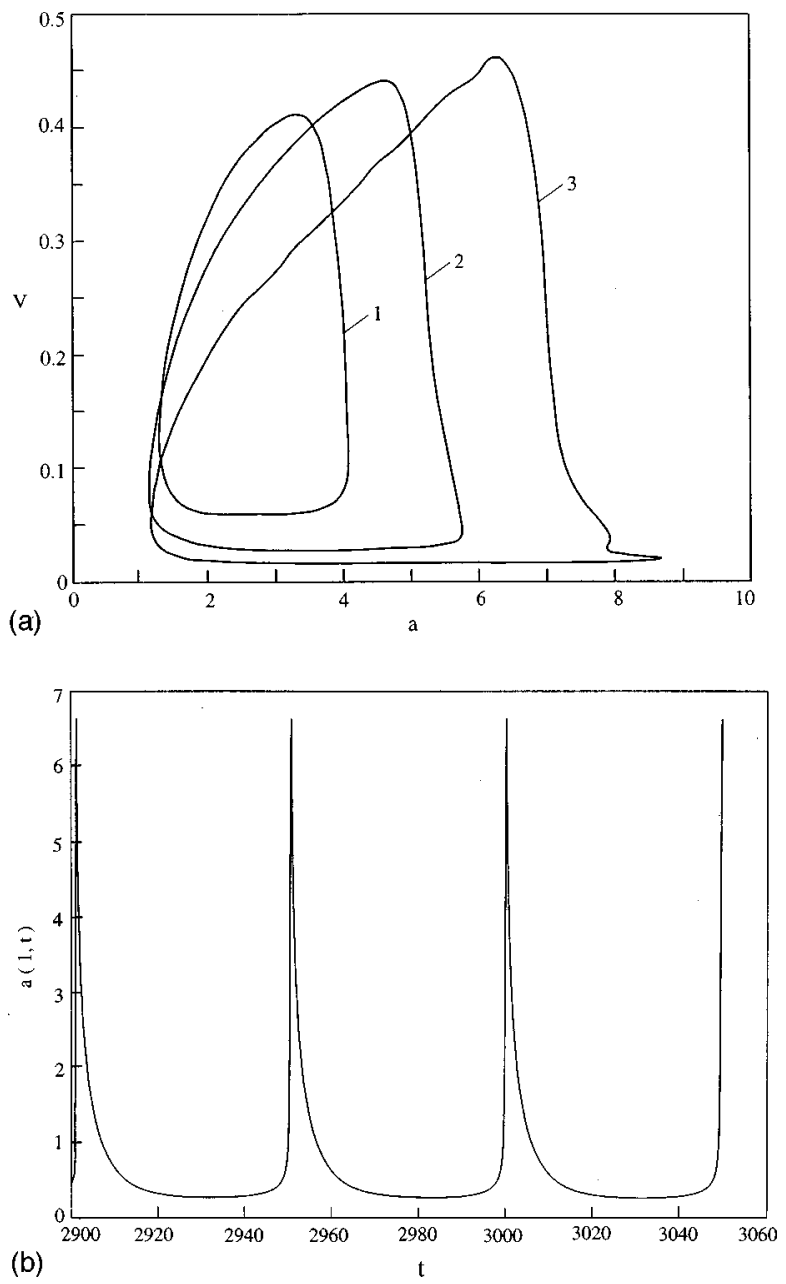

FIG. 1. (a) The phase plane describing the fiber midlength $x=0.5$. Limitcycle behavior (draw resonance) at (1) $E=28$, (2) -50 , and (3) -100 . No forcing, $\delta=0$. (b) The profile $a(1, t)$ corresponding to $E=100, \delta=0$.

boundary conditions (16) and (17) at $E>20.22$. This means that we introduce a local fluctuation upstream and watch it grow downstream.

\section{NUMERICAL SOLUTION AND BIFURCATION STRUCTURE OF THE NONLINEAR PROBLEM}

In this section we present the numerical results obtained from simulation of the continuity Eq. (1) and the full momentum Eq. (3) with time dependent boundary conditions (at $x=0)(16)$ and (17). A modified version of the algorithm of Refs. 23, 26, and 30 was used in the present work. The initial-value problem (1) and (3) was solved by a direct implicit scheme of the Crank-Nicholson-type. Nonsymmetric approximations were used for $\partial a / \partial x$, and symmetric ones for $\partial V / \partial x$ and $\partial^{2} V / \partial x^{2}$, with the accuracy $O\left(\Delta x^{2}\right)$. The time derivatives $\partial a / \partial t$ and $\partial V / \partial t$ were approximated using a two-layer scheme with accuracy $O(\Delta t)$. Some more details on the numerical implementation may be found in Ref. 23 and 26. In all the calculations the dimensionless parameters had the following values: $\mathrm{Re}=7.5 \cdot 10^{-6}, \mathrm{We}=0.15 \mathrm{Fr}$ $=0.051, L / a_{0}=30$, and thus $C_{1}=7.5 \cdot 10^{-5}, C_{2}=0.0441$, and $C_{3}=O\left(10^{-3}\right)$. The values of $C_{3}$ correspond to the values of $E$ which varied in the range $19<E \leqslant 100$ from one

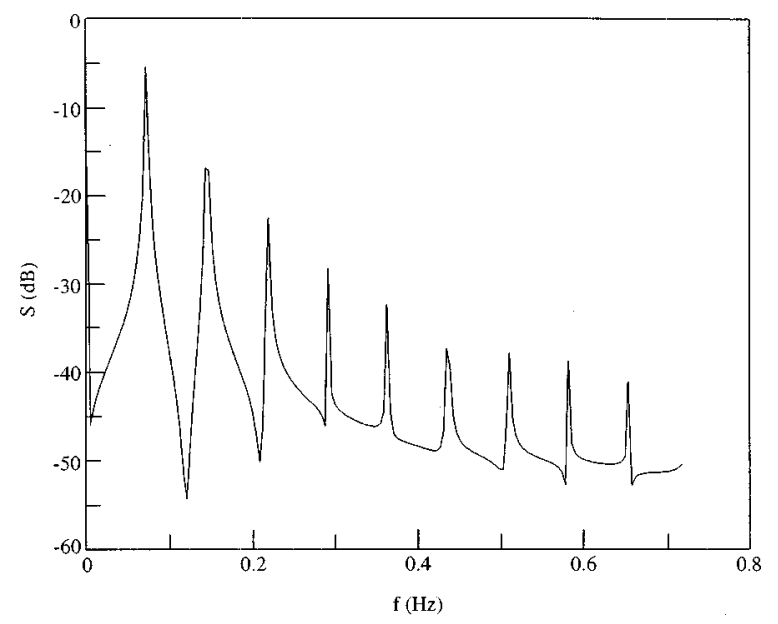

FIG. 2. Power spectrum that corresponds to the limit cycle at $E=28$ [curve 1 in Fig. 1(a)].

numerical experiment to another. Note that at the fixed values of the other parameters such a variation in $E$ means that the input velocity varied from one numerical experiment to another.

\section{A. The unperturbed problem $(\delta=0)$}

The numerical simulations of the full set of the governing Eqs. (1) and (3) reveal the draw resonance instability and transition to periodic flow in an unperturbed problem at the value of the draw ratio close to $E=20.22$ which was obtained via the linear analysis in Sec. III, where the secondary effects were neglected. This supports our expectations based on the physical estimates of a real drawing process given in Sec. II, that the inertial, gravitational and surface tension forces are not important in the present case. Similar conclusions for the same range of the parameters were described in Refs. 23 and 26.

As noted in the previous section for $E>20.22$, a selfsustained limit cycle evolves as the steady fixed point solution loses its stability. This limit cycle is stable and grows in size with growing values of $E$ as is depicted in Fig. 1(a), which illustrates the fiber midlength phase plane $(a(0.5, t)$, $V(0.5, t))$. The degree of complexity (loss of circularity) of the phase plane is apparent for large $E$. The profile $a(1, t)$ at $E=100$ is shown in Fig. 1(b). Note that the degree of solution nonlinearity is also depicted by the amplitudes of the harmonics in the power spectra in Figs. 2 and 3 that correspond to limit cycles 1 and 3 of Fig. 1. We summarize the change in radius $\Delta a=a_{\max }-a_{\min }$ and self-sustained limit cycle frequency $\omega_{l c}$ in Fig. 4 . We note that the degree of nonlinearity is portrayed by the difference between the linear frequency approximation (i.e., the complex part of the eigenvalues $\Lambda_{i}$ in Table I) and those obtained from the nonlinear simulation, $\omega_{l c} \cdot{ }^{23}$

\section{B. The perturbed problem $(\delta>0)$}

The fundamental solution of the periodically perturbed self-excited limit cycle is a quasiperiodic torus that is generated for an exciting frequency $\omega$ that is incommensurate with that of the self-sustained limit cycle. This solution is por- 


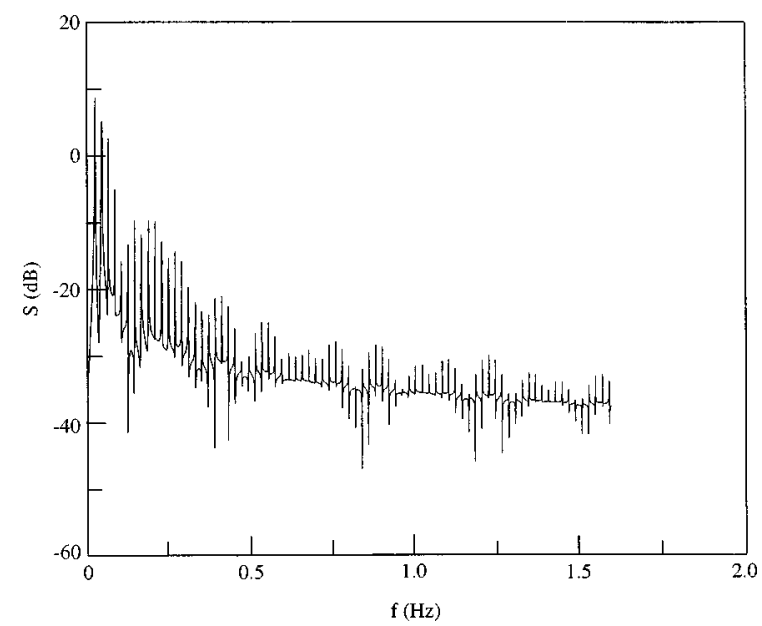

FIG. 3. Power spectrum that corresponds to the limit cycle at $E=100$ [curve 3 in Fig. 1(a)].

trayed in Fig. 5, where we combine the phase plane containing the unperturbed limit cycle 1 with the Poincare map $(a(0.5, t), V(0.5, t))$ corresponding to the excited flow stroboscopically sampled $(t=\omega T)$ each forcing frequency $\omega$. Note that the sampled points follow the contours of the unperturbed limit cycle phase plane. It is emphasized that here and hereinafter the qualitative character of the Poincare maps does not change irrespective of the choice of the cross section, where $a$ and $V$ were sampled. As predicted by theory (cf. Ref. 25), the forced fundamental quasiperiodic solution is replaced by a periodic (mode-locked) ultrasubharmonic ( $m \omega \approx n \omega_{l c} ; m$ and $n$ are integers) solution above a certain threshold controlled by the system parameters. A period doubled solution (defined by two Poincaré points) is depicted in Fig. 6 for excitation near twice that of the unperturbed limit cycle $\left(\omega=1, \omega_{l c} \approx 0.42\right)$ for the case of $E=30$. An increase in $E$ with constant $\omega$ (or alternatively change in $\omega$ for $E=$ const $)$ yields a period-four subharmonic for $E=50\left(\omega_{l c}\right.$ $\approx 0.24)$. The periodic mode-locked solutions are organized in the stability parameter space $(\omega, \delta, E)$ in the form of wedges (or Arnold tongues obtained for a circle map ${ }^{31}$ ) separating the quasiperiodic solutions. For increasing forcing $(\delta)$ the distinct structure is broken and complex periodic and

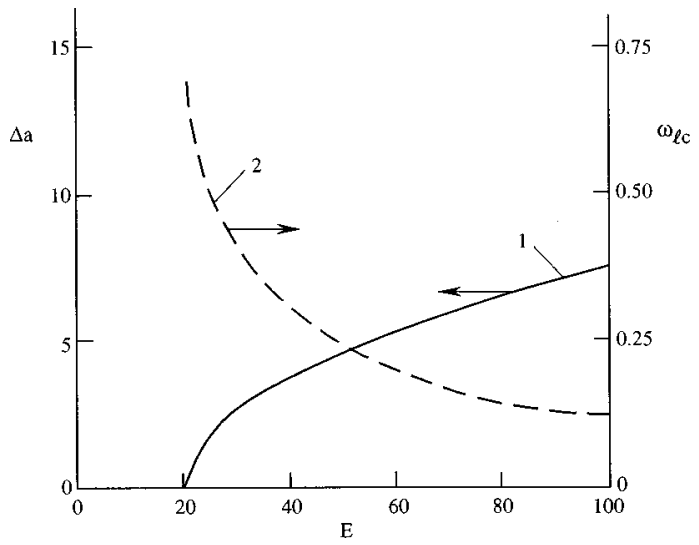

FIG. 4. Limit cycle radius $\Delta a$ and frequency $\omega_{l c}$ as functions of the draw ratio. No forcing, $\delta=0$.

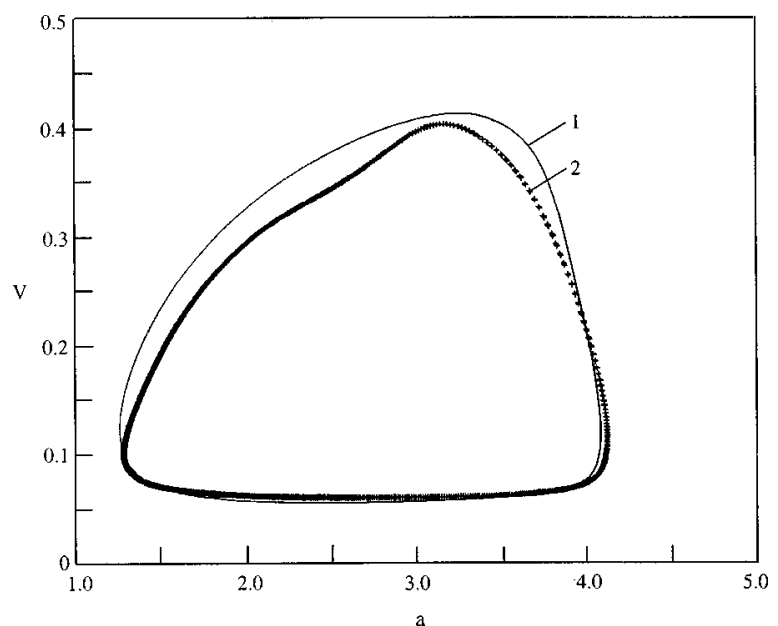

FIG. 5. The phase plane of the unperturbed flow containing limit cycle (1) combined with the phase plane (2), and the Poincare map of the excited flow $(\delta=0.01, \omega=1)$ depicting two sampled points. $E=30, x=0.5$.

aperiodic solutions coexist with strange attractors. Two examples of strange attractors are depicted $(\delta=0.1, \omega=1)$ in Figs. 7 and 8 for $E=30$ and $E=50$, respectively, and the corresponding fiber profiles $a(1, t)$ in Figs. 7(b) and 8(b). Note the degree of complexity increases as the solutions fill more of the system phase space with increasing nonlinearity. Furthermore, the threshold of stability of the mode-locked solutions decreases with increasing $E$.

It is emphasized that, in spite of the temporal complexity of the results shown in Figs. 7(b) and 8(b), the shapes of the whole fiber in the drawing zone do not differ too much from those corresponding to draw resonance (cf., for example, Fig. 6 in Ref. 23). Namely, the perturbation waves propagating over the threadline represent themselves very long waves with the wavelength of the order of the whole drawing zone. This observation allows us to conclude a posteriori that the solutions obtained do not contradict the assumptions of the quasi-one-dimensional approximation under which the governing equations are valid. This observation also allows us to

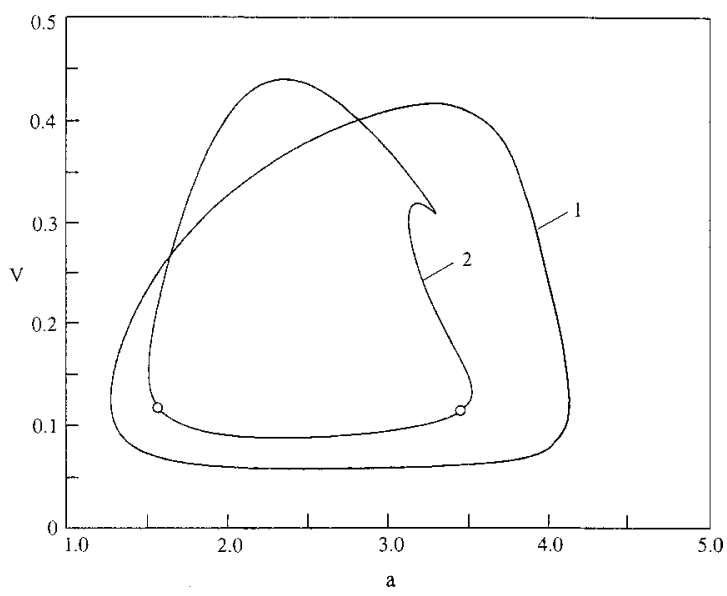

FIG. 6. The phase plane of the limit cycle 1 of the unperturbed flow, and the phase plane of the excited flow $(\delta=0.04, \omega=1)$ combined with the Poincare map of the latter containing two points (symbols) which corresponds to period doubling. $E=30 ; x=0.5$. 

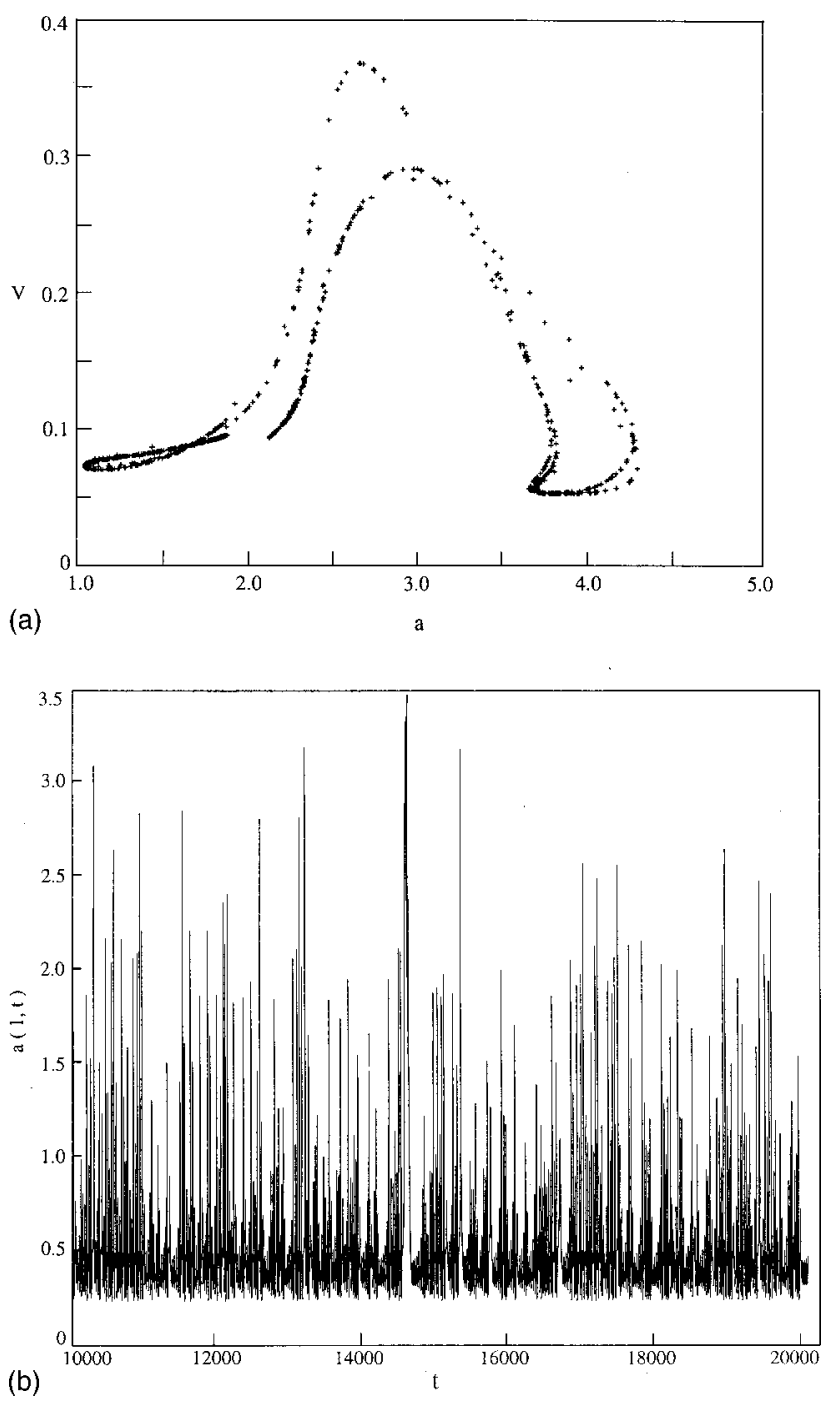

FIG. 7. (a) Strange attractor for $\delta=0.13, \omega=1$, and $E=30$. The Poincaré map was sampled at $x=0.5$. (b) The corresponding fiber profile $a(1, t)$.

conclude that the irregular behavior depicted in Figs. 7(b) and 8 (b) consists of spatially coherent temporal chaos. Whether any additional routes to chaos can emerge in a fully three-dimensional case, where intermediate length scales violating the quasi-one-dimensional assumptions can appear, is (as in many other cases known in literature) an open question at present, since such three-dimensional calculations challenge the current tools. Moreover, the fact that even the problems solvable numerically without simplifications in the governing equations are inevitably subject to truncation errors affecting the bifurcation transfer energy to lower scales, stresses the idea that "true" chaotic motions may be, in principle, incomputable in their full detail. ${ }^{32}$

\section{The bifurcation structure and routes to chaos}

As noted above, the solution structure is organized in a classical Arnold tongue wedges which overlap for large perturbation. We identify two distinct mechanisms for evolution of the fundamental solution to chaos; (i) a smooth perioddoubling transition of a fundamental periodic solution $\left(2^{n} T\right)$. This bifurcation emerges within the mode-locked

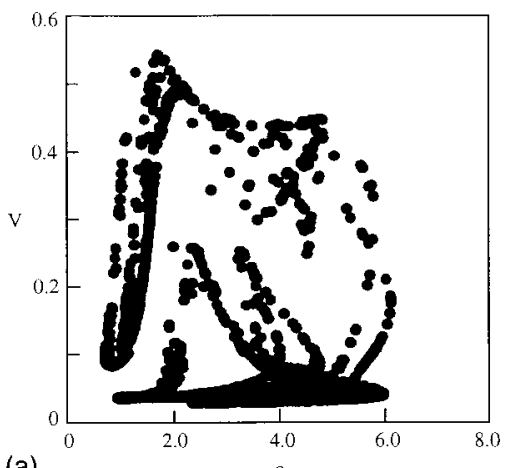

(a)

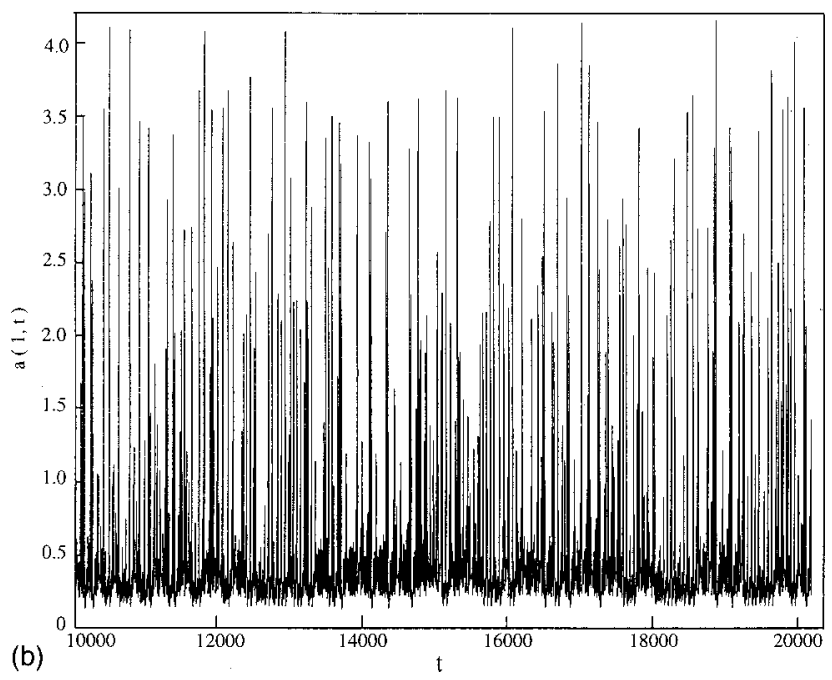

FIG. 8. (a) Strange attractor for $\delta=0.1, \omega=1$, and $E=50$. The Poincaré map was sampled at $x=0.5$. (b) The corresponding fiber profile $a(1, t)$.

wedge for constant $E, \omega$ and increasing forcing $\delta$; (ii) an abrupt explosion from the fundamental quasiperiodic solution to a chaotic one. For relatively small $E$ (e.g., $E=30$ ) the chaotic attractor (Fig. 7) is confined by forward $(4 T, 8 T, \ldots)$ and reverse $(8 T, 4 T, 2 T)$ period doubling bifurcations (Fig. 9 ). With increasing $E$, additional solutions in the form of quasiperiodic tori (Fig. 10 for $E=50$ ) appear and break the forward period-doubling bifurcation sequence within the wedge with a strange attractor (Fig. 11). These additional quasiperiodic solutions correspond to the domain $Q^{s}$ in Fig. $12(E=50)$ which is absent in Fig. $9(E=30)$. Also the

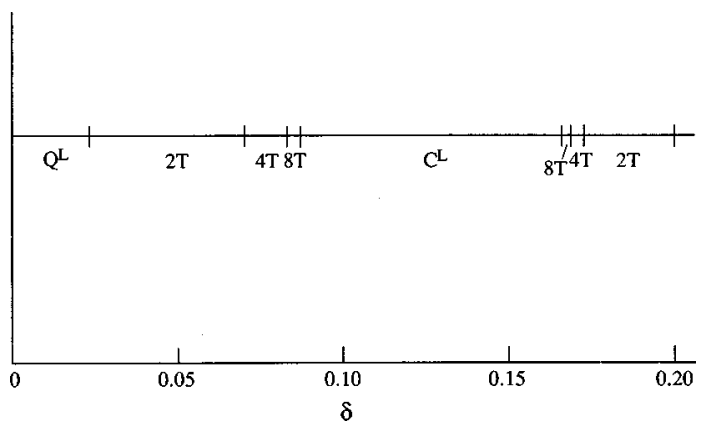

FIG. 9. Forward period doubling route to chaos, and reverse period doubling after the strange attractor disappears. $E=30 . Q^{L}$ corresponds to quasiperiodic solutions, $2^{n} T$ to period doubling routes and $C^{L}$ to the domain of the strange attractor. 


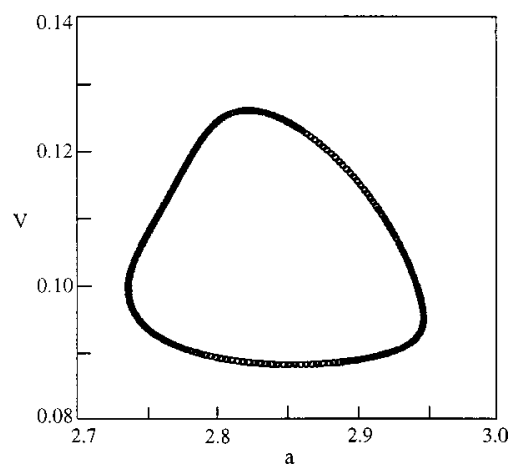

FIG. 10. The Poincaré map at $E=50$ for a "small" quasiperiodic solution. Exciting amplitude and frequency $\delta=0.03$ and $\omega=1$, respectively. Sampled at $x=0.5$.

domain of the smaller strange attractor that occupies a smaller part of the phase space (see Fig. 11 and domain $C^{S}$ in Fig. 12) was not revealed for smaller $E$ (cf. Fig. 9). After the small strange attractor disappears the forward period doubling sequence reveals a large strange attractor (domain $C^{L}$ in Fig. 12) similar to that found for $E=30$ (cf. Fig. 9). After all this strange attractor is broken by a reverse period doubling route (Fig. 12).

The physical mechanism that drives the boundary-value problem considered is a specific form of periodic boundary excitation of an (unperturbed) stable flow that exhibits a fundamental periodic state. The latter is the draw resonance in the present case.

This mechanism consists of periodic forcing in the form of (local) parametric excitation of a coupled set of self excited oscillators (e.g., forced van-der Pol oscillators) that reveals both (i) the global bifurcation phenomena of interchanging quasiperiodic tori with periodic mode-locked (ultrasubharmonic) oscillations for an incommensurate/ commensurate frequency ratio or irrational/rational rotation (cf. Ref. 33); (ii) period-doubling cascade of a fundamental mode-locked solution. Both of the noted phenomena continue to evolve (with changing parameters) and lose their stability culminating with (several) distinct types of chaotic strange attractors.

The parametric excitation of a continuum dynamical system via periodic boundary excitation yields the fundamental

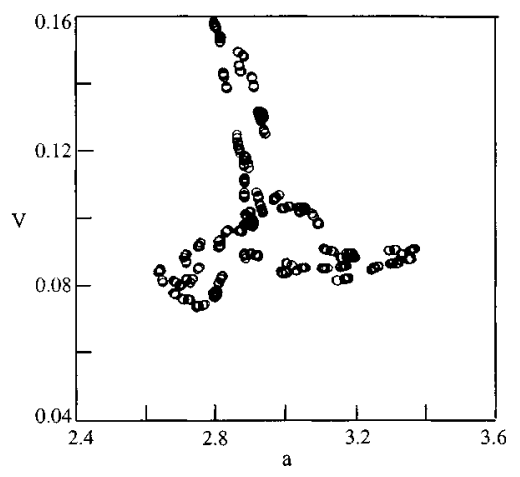

FIG. 11. The Poincaré map at $E=50$ for a "small" strange attractor. Exciting amplitude and frequency $\delta=0.04$ and $\omega=1$, respectively. Sampled at $x=0.5$.

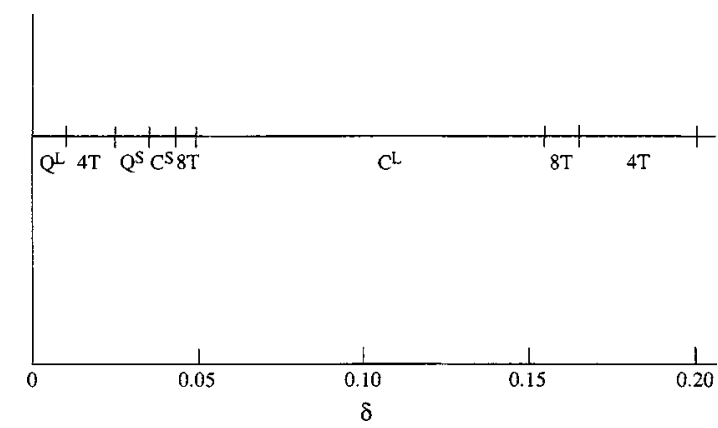

FIG. 12. The bifurcation structure at $E=50 . Q^{L}$ indicates quasiperiodic solutions corresponding to a "large spot" on the Poincaré map similar to that of Fig. 9. $2^{n} T$, the domains of period doubling. $Q^{S}$ indicates the domain of the quasiperiodic solutions corresponding to a "small spot." $C^{s}$ indicates the domain of a small strange attractor exemplified, e.g., by Fig. 11. $C^{L}$ indicates the domain of a large strange attractor like that of Figs. 7 and 9.

Floquet structure (for weak forcing) that demonstrates a period doubling instability. ${ }^{34}$ However, the existence of a "hidden" frequency (of the unperturbed problem) that interacts with that of the excitation brings forth the quasiperiodic and mode-locked attractors confining the period doubling cascade in a limited parameter space.

Finally, we note that additional forms of periodic boundary excitation may not bring forth the phenomena noted as the parametrically excited forcing may not interact directly with the fundamental (unperturbed) periodic solution. This can be seen in the direct (external excitation, Ref. 35) and indirect (parametric via vertical or horizontal boundary excitation) oscillations of an inverted pendulum which yield a "kaleidoscope of different stable oscillations". ${ }^{36}$ This explains why in an additional series of computations we have not been able to obtain an aperiodic response for the drawn fiber on the fluctuations of the input and output velocity alone. These perturbations in the studied range of parameters simply do not lead to bifurcations towards chaos, which, however, does not preclude them to generate an aperiodic response for other parameter sets (which may be, however, physically unrealistic). We can conclude that fluctuations in the input radius can be the main source of chaos.

\section{CONCLUDING REMARKS}

The cross section of Newtonian isothermal fibers may vary nonperiodically (quasiperiodically or chaotically) at draw ratio $E$ about 30 and more under the conditions of periodic variations of the input cross section. The route to chaos may be smooth, via period doubling, or explosive, via abrupt disappearance of quasiperiodic solutions. As a result of period doubling or abrupt explosion of quasiperiodic solutions a strange attractor sets in. We note that this irregular behavior, which consists of spatially coherent temporal chaos in the quasi-one-dimensional fiber model, indicates to corresponding three-dimensional instabilities but that further work (e.g., experimental and numerical) is required to resolve whether the strange attractors found correspond to spatially coherent temporally chaos in a fully three-dimensional model. 
The model problem considered mimics glass fiber drawing processes. In the direct-melt processes fibers are formed by drawing molten glass from heated furnaces and the upstream perturbations can set in mainly due to the thermal inertia of the furnace. In the fiber drawing from solid preforms a nonuniform cross-sectional radius of the latter can be a source of the upstream perturbations. The results obtained point out on a possibility that drawn fibers may be perturbed nonperiodically. The experimental data of Ref. 24 and references therein show that this, indeed, can take place in glass fiber drawing.

${ }^{1}$ R. F. Caroselli, "Glass textile fibers," in Man-Made Fibers, Science, and Technology, edited by H. F. Mark, S. M. Atlas, and E. Cernia (Interscience, New York, 1968), Vol. 3, pp. 425-454.

${ }^{2}$ J. G. Mohr and W. P. Rowe, Fiber Glass (Van Nostrand Reinhold, New York, 1978)

${ }^{3}$ J. A. Burgman, "Liquid glass jets in the forming of continuous glass fibers," Glass Technol. 11, 110 (1970)

${ }^{4}$ S. M. Oh, "Cooling rates of optical fibers during drawing," Ceram. Bull. 58, 1108 (1979)

${ }^{5}$ I. P. Kaminow, "Polarization-maintaining fibers," in Proceedings of the First International Conference at MIT, 1981, p. 169.

${ }^{6}$ J. Doupovec and A. L. Yarin, "Nonsymmetrical modified chemical vapor deposition (N-MCVD) process," J. Lightwave Technol. 9, 695 (1991).

${ }^{7}$ J. R. A. Pearson and M. A. Matovich, "Spinning a molten threadline. Stability,'” Ind. Eng. Chem. Fundam. 8, 605 (1969).

${ }^{8} \mathrm{H}$. Ishihara and S. Kase, "Studies on melt spinning. V. Draw resonance as a limit cycle,'” J. Appl. Polym. Sci. 19, 557 (1975).

${ }^{9}$ F. T. Geyling and G. M. Homsy, "Extensional instabilities of the glass fiber drawing process," Glass Technol. 21, 95 (1980).

${ }^{10}$ A. L. Yarin, "On generation of self-sustained oscillations during fiber formation," J. Appl. Math. Mech. 47, 59 (1983).

${ }^{11}$ W. W. Schultz, A. Zebib, S. H. Davis, and Y. Lee, "Nonlinear stability of Newtonian fibers," J. Fluid Mech. 149, 455 (1984).

${ }^{12}$ Y. T. Shah and J. R. A. Pearson, "On the stability of nonisothermal fiber spinning," Ind. Eng. Chem. Fundam. 11, 145 (1972).

${ }^{13}$ Y. T. Shah and J. R. A. Pearson, "On the stability of nonisothermal fibre spinning: General case," Ind. Eng. Chem. Fundam. 11, 150 (1972).

${ }^{14} \mathrm{~A}$. L. Yarin, "Effect of heat removal on nonsteady regimes of fiber formation," J. Eng. Phys. 50, 569 (1986).

${ }^{15}$ A. L. Yarin, Free Liquid Jets and Films: Hydrodynamics and Rheology (Longman, copublished with Wiley, Harlow and New York, 1993).

${ }^{16}$ L. R. Glicksman, "The dynamics of a heated free jet of variable viscosity liquid at low Reynolds numbers," Trans. ASME, J. Basic Eng., Series D 90, 343 (1968).
${ }^{17}$ B. P. Huynh and R. I. Tanner, "Study of the non isothermal glass fiber drawing process," Rheol. Acta 22, 482 (1983).

${ }^{18}$ C. B. Weinberger, G. F. Cruz-Saenz, and G. J. Donnelly, "'Onset of draw resonance during isothermal melt spinning: A comparison between measurements and predictions," AIChE. J. 22, 441 (1976).

${ }^{19}$ V. M. Entov and A. L. Yarin, "The dynamics of thin liquid jets in air," J. Fluid Mech. 140, 91 (1984)

${ }^{20}$ D. W. Bousfield, R. Keunings, G. Marrucci, and M. M. Denn, "Nonlinear analysis of the surface tension driven breakup of viscoelastic filaments," J. Non-Newtonian Fluid Mech. 21, 79 (1986).

${ }^{21}$ S. E. Bechtel, J. Z. Cao, and M. G. Forest, "Practical application of a higher order perturbation theory for slender viscoelastic jets and fibers," $\mathrm{J}$. Non-Newtonian Fluid Mech. 41, 201 (1992).

${ }^{22}$ S. E. Bechtel, J. Z. Cao, and M. G. Forest, "Illustration of an optimization procedure for fiber-spinning operating conditions: Maximum draw ratio under a Maxwell thin-filament model," J. Rheol. 37, 237 (1993).

${ }^{23}$ A. L. Yarin, P. Gospodinov, and V. Roussinov, "Stability loss and sensitivity in hollow fiber drawing," Phys. Fluids 6, 1454 (1994).

${ }^{24}$ N. I. Tyushkevich, A. S. Kras'ko, A. A. Chepurkin, O. P. Shiman, T. O. Kozello, and N. A. Ananich, "Study of the unevenness of glass fiber by statistical methods," Glass and Ceramics 27, 95 (1970).

${ }^{25}$ J. A. Glazier and A. Libchaber, "Quasiperiodicity and dynamical systems: An experimentalist's view," IEEE Trans. Circuits Syst. 35, 790 (1988).

${ }^{26} \mathrm{P}$. Gospodinov and V. Roussinov, "Nonlinear instability during the isothermal drawing of optical fibers," Int. J. Multiphase Flow 19, 1153 (1993).

${ }^{27}$ S. P. Radev, P. N. Gospodinov, V. M. Roussinov, and A. L. Yarin, "Method of measurement of the activation energy of viscous glass flow," Patent N. 86349-47316, Bulgaria, 1988.

${ }^{28}$ A. Ziabicki, Fundamentals of Fiber Formation (Wiley, London, 1976).

${ }^{29}$ High-Speed Fiber Spinning, edited by A. Ziabicki and H. Kawai (Wiley, New York, 1985).

${ }^{30} \mathrm{P}$. Gospodinov and A. L. Yarin, "Draw resonance of optical microcapillaries in non-isothermal drawing," Int. J. Multiphase Flow 23, 967 (1997).

${ }^{31}$ A. J. Lichtenberg and M. A. Lieberman, Regular and Chaotic Dynamics (Springer, Berlin, 1992)

${ }^{32}$ A. L. Yarin, O. Gottlieb, and I. V. Roisman, "Chaotic rotation of triaxial ellipsoids in simple shear flow,"' J. Fluid Mech. 340, 83 (1997).

${ }^{33}$ J. Guckenheimer and P. Holmes, Nonlinear Oscillations, Dynamical Systems, and Bifurcations of Vector Fields (Springer, New York, 1983).

${ }^{34}$ P. G. Drazin and W. H. Reid, Hydrodynamic Stability (Cambridge University Press, Cambridge, 1995).

${ }^{35}$ J. Miles, "Direclty forced oscillations of an inverted pendulum," Phys. Lett. A 133, 295 (1988)

${ }^{36} \mathrm{P}$. J. Bryant and J. Miles, "On a periodically forced weakly damped pendulum,” J. Aust. Math. Soc. B, Appl. Math. 32, 1 (1990). 